\title{
Abstracts From the NCCN 2020 Virtual Annual Conference
}

\begin{abstract}
The following abstracts were accepted for presentation at the NCCN 2020 Annual Conference. Additional abstracts are available at JNCCN.org and posters are available at NCCN.org.
\end{abstract}

J Natl Compr Canc Netw 2020;18(7.5):919-933 doi: 10.6004/jnccn.2020.5002

\section{YOUNG INVESTIGATOR AWARDS}

YIA20-001: Targeting TGF- $\beta$ in Ovarian Cancer Results in Decreased Tumor Burden and Improved T Cell Immune Response

Rebecca Arend, MD; Brandon M. Roane, MD;

Selene Mesa-Perez, PhD; Whitney N. Goldsberry, MD;

Ashwini Katre, MS; Michael J. Birrer, MD; and Lisa A. Norian, PhD

University of Alabama at Birmingham, Birmingham, AL

Background: Increased transforming growth factor beta (TGF- $\beta$ ) is associated with poorer prognosis in advanced stage endometrial carcinoma (EOC). The immunosuppressive effects of this family of proteins promotes tumor progression and diminishes response to therapy. The objective of this study was to evaluate the effects of antiTGF- $\beta$ therapy in an immunocompetent ovarian cancer mouse model. Methods: ID8-p53-/- cells were injected intraperitoneally (I.P.) into C57BL/6 mice to establish our syngeneic ovarian cancer model. An intraperitoneal monoclonal antibody targeting all three TGF- $\beta$ ligands was used as our anti-TGF- $\beta$ therapy. Timing of anti-TGF- $\beta$ therapy had three different treatment regimens. After 42 days of tumor challenge, ascites and omenta were harvested and weighed for tumor burden and changes in $\mathrm{T}$ cell response were measured using flow cytometry to calculate the CD8 to regulatory $\mathrm{T}$ cell (Tregs) ratio. Results: Treatment with anti-TGF- $\beta$ therapy every other day beginning 7 days following tumor challenge resulted in decreased ascites volume $(4.1 \mathrm{~mL}$ vs $0.7 \mathrm{~mL} ; \mathrm{p}<0.001)$ and improvement in CD8: Treg ratio ( 0.37 vs $2.5 ; \mathrm{p}=0.02$ ). However, there was no difference seen in the omental weights comparing treated and untreated mice. A single dose of anti-TGF- $\beta$ therapy one day prior to 42 days of tumor challenge and with no additional therapy resulted in a similar reduction of ascites volume $(2.7 \mathrm{~mL}$ vs. $0.67 \mathrm{~mL}$ $\mathrm{p}=0.0024)$ and increased CD8 : Tregs ratio (0.36 vs 1.49 ; $\mathrm{p}=0.007)$, while also significantly reducing omental weight $(114.9 \mathrm{mg}$ vs $93.4 \mathrm{mg} ; \mathrm{p}=0.017)$. When the two regimens were combined - one dose prior to tumor cell injection followed by a dose every other day for 42 days, there was a significant reduction in ascites volume $(2.24 \mathrm{~mL}$ vs $0.69 \mathrm{~mL} ; \mathrm{p}=0.0025)$ and omental weight (124.9mg vs $84.3 \mathrm{mg} \mathrm{p}=0.004)$ and favorable changes in
CD8 : Tregs ratio ( 0.357 vs. $1.49 ; \mathrm{p}=<0.001$ ). Furthermore, this regimen demonstrated prolonged overall survival, with median survival of 70 days in treated mice compared to 57.5 days in untreated mice. Conclusions: We show that inhibition of TGF- $\beta$ with a monoclonal antibody results in reduced ascites production as well as favorable changes in the $\mathrm{T}$ cell profile. When treatment is introduced prior to tumor challenge, there is a reduction in tumor burden. These results suggest the potential for using anti-TGF- $\beta$ therapy in the maintenance setting or in conjunction with immunotherapy in ovarian cancer patients.

YIA20-002: Racial Differences in Clinical Histopathological Features and Survival of Early- and Average-Onset

\section{Colorectal Cancer}

Hanyu Chen, MDª, ; Jie Liu, MDa; Xiaoyu Zong, MPHa;

Ai Zhang, MPHa; Jennifer Tappenden, MFAa; Thomas Walsh, BSa; Deyali Chatterjee, MDa; Ryan Courtney Fields, MDa;

Graham Andrew Colditz, MD, DrPHa,c; and Yin Cao, MPH, ScD a,c a'Washington University School of Medicine, St. Louis, MO; 'bey Laboratory of Precision Diagnosis and Treatment of Gastrointestinal Tumors, Ministry of Education, The First Affiliated Hospital of China Medical University, Liaoning, P.R. China; 'Siteman Cancer Center, Washington University School of Medicine, St Louis, MO

Background: The rise of early-onset colorectal cancer (CRC) among non-Hispanic whites (NHW) compared to non-Hispanic blacks (NHB) points to race-specific etiology. Racial differences in clinical characteristics and survival differences for early-onset CRCs remain to be explored. Methods: Data from 4,012 CRC patients treated at the Siteman Cancer Center (2000-2015) was retrieved. We compared patient (insurance, comorbidities, family history of CRC) and clinical characteristics (site, stage, grade, histology), and treatment/surgical outcomes (surgery/radiation/chemotherapy, surgical approach, surgical margins, retrieved lymph nodes) according to race (NHB vs NHW) within 945 early-onset $(<50 \mathrm{yr}$ ) and 1,580 average-onset CRC cases ( $\geq 65 \mathrm{yr}$ ), respectively. We then evaluated racial differences in 5-year survival. Cox models were used to estimate hazard ratios (HR) and 95\% confidence intervals (CIs). Results: Compared to early-onset CRCs among NHW, NHB cases were more likely to present at the proximal (39\% vs $23 \%$ ) and distal colon ( $39 \%$ vs $33 \%$ ) but less likely to be at the rectum 
(23\% vs $44 \%)(\mathrm{P}<0.001)$, while other clinical features were similar. Early-onset NHB CRC patients were less likely to have commercial insurance ( $47 \%$ vs $80 \%$ ), more likely to enroll in Medicaid (32\% vs $12 \%$ ) or Medicare $(9.4 \%$ vs $3.5 \%)(\mathrm{P}<0.001)$, and present with a greater comorbidity score $(23 \%$ vs $16 \%)(\mathrm{P}=0.048)$. Five-year survival (crude rate $53 \%$ vs $61 \%$ ) was slightly worse for early-onset NHB compared to NHW cases after adjusting for age, sex, year of diagnosis, and family history ( $\mathrm{HR}=1.26$; 95\% CI 0.97-1.64), but the differences diminished after further adjustment for insurance, comorbidities, clinical features, treatment, and surgical outcomes ( $\mathrm{HR}=1.12$; 95\% CI 0.83-1.50). Interestingly, except for advanced stage (IV: $34 \%$ vs $16 \%$ ), minimal differences were observed for site/grade/ histology when comparing early- vs average-onset NHB cases $(\mathrm{P}>0.05)$. In contrast, for NHW cases, in addition to advanced stages (IV: $33 \%$ vs $15 \%$; $\mathrm{P}<0.001$ ), early-onset CRCs are also more likely to be at the rectum ( $44 \%$ vs $38 \%$ ) $(\mathrm{P}<0.001)$ and show high grade of differentiation $(27 \%$ vs $22 \%$; $<0.001$ ) compared to NHW CRCs of average-onset. Conclusions: Our findings on differential tumor sites for early-onset NHB vs NHW CRCs as well as more advanced clinical characteristics of early-onset NHW cases compared to those of late-onset together call for deep molecular profiling of both early-onset NHB and NHW CRCs.

\footnotetext{
YIA20-003: CD229 CART Cells Eliminate Multiple Myeloma and Tumor Propagating Cells but Show Limited Targeting of Normal T Cells

Tim Luetkens, MDa; Sabarinath Venniyil Radhakrishnan, MDa;

Sandra D. Scherer, PhDa; Patricia Davis, PhD';

Erica R. Vander Mause, PhDa; Michael L. Olson, $\mathrm{PhD}^{\mathrm{a}}$;

Sara Yousef, PhDa; Jens Panse, MDc; Yasmina Abdiche, PhD;

K. David Li, MD ${ }^{\text {; }}$ Rodney R. Miles, MD' ${ }^{\text {; }}$ William Matsui, MD ${ }^{\mathrm{e}}$;

Alana L. Welm, PhDa; and Djordje Atanackovic, MDa

aHuntsman Cancer Institute, University of Utah, Salt Lake City, UT;

bUniversity of Utah and ARUP Laboratories, Salt Lake City, UT;

cUniversity Hospital RWTH Aachen, Aachen, Germany; ${ }^{d}$ Carterra Inc.,

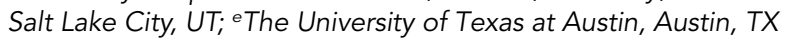

Background: The SLAM receptor CD229/LY9 is strongly expressed on multiple myeloma (MM) cells and is required for their growth, rendering it a potential target for the treatment of MM. Methods: We used antibody phage display to develop the first fully human antibody targeting CD229, clone 2D3. Using various screening assays, as well as biochemical characterization and functional assays, we evaluated this antibody and T cells expressing a chimeric antigen receptor (CAR) based on this antibody. Lowaffinity antibodies were generated using a single site saturation variant library and characterized using highthroughput surface plasmon resonance. Results: 2D3 binds strongly to the malignant plasma cells from 20/20 analyzed patients with newly diagnosed and relapsedrefractory MM as well as normal memory $B$ and $T$ cells but not activated $\mathrm{T}$ cells due to the rapid downregulation of
CD229 during T cell activation allowing efficient CD229 CAR T cell production. CD229 CAR T cells specifically kill CD229-expressing cells and show potent killing of MM cell lines and primary plasma cell leukemia cells. In contrast to BCMA CAR T cells, CD229 CAR T cells also eliminate memory B cells, a potential reservoir of clonotypic MM precursors. We further observed significantly reduced colony formation by CD34neg bone marrow cells from patients with MM after treatment with CD229 CAR T cells compared to BCMA CAR T cells, indicating more efficient targeting of tumor propagating cells. In vivo treatment with a single dose of CD229 CAR T cells resulted in tumor eradication or delayed tumor growth and significantly prolonged survival. While we observed that CD229 CAR T cells target resting CD229high $\mathrm{T}$ cells, they spared CD229neg/low T cells. CD229neg/low T cells show the same phenotype as CD229high T cells and conventional effector functions in response to common pathogens. To further reduce the targeting of normal $\mathrm{T}$ cells, we generated low-affinity CD229 antibodies and show that CAR T cells based on the these antibodies have increased selectivity for MM cells over normal T cells. Conclusions: CD229 CAR $T$ cells can be manufactured efficiently and are highly active against MM, including tumor propagating cells. CD229 CAR T cells show limited fratricide during CAR $\mathrm{T}$ cell production, spare a functional $\mathrm{CD} 229 n e g /$ low $\mathrm{T}$ cell population, and reducing antibody affinity further enhances their selectivity for MM. CAR T cells targeting CD229 are a promising new approach for the treatment of MM.

YIA20-004: Altering the Local Immune Landscape in Lung Cancer to Improve Checkpoint Inhibitor Therapy

Nancy Danielle Ebelt, BS, PhD; Edith Zuniga, BS;

and Edwin Ramos Manuel, PhD

Beckman Research Institute of the City of Hope, Duarte, CA

Non-small cell lung cancer (NSCLC) is the leading cause of cancer-related deaths worldwide. Immune checkpoint blockers (ICBs) composed of antibodies targeting programmed death 1 (anti-PD1) and cytotoxic T-lymphocyte-associated protein 4 (anti-CTLA-4) have shown great promise in treating NSCLC, but these responses are not universal among patients and are often met with high risk for developing resistance. A key feature of NSCLC is a tumor infiltrate dominated by suppressive immune subsets such as regulatory T cells, NK cells, macrophages and neutrophils, which have been associated with significant immune suppression and overall poor survival. While the preponderance of infiltrating suppressive immune subsets likely contributes to checkpoint inhibitor resistance, new studies highlight how reprogramming the immune microenvironment can overcome resistance mechanisms to immunotherapy. Our central hypothesis is that by enhancing the overall anti-tumor immune 
phenotype within NSCLC tumors, we can enhance response rates to ICBs. To convert and/or expand antitumor subsets within an ICB non-responsive model of NSCLC, we utilized our Salmonella typhimurium -based platform targeting indoleamine 2,3-dioxygenase (shIDO-ST), which we have confirmed is specifically engulfed by neutrophils and induces hyperactivation of their antitumor (N1) functions. Following shIDO-ST treatment, we observed broader changes in intratumoral immune repertoire and phenotype, in addition to N1 neutrophils, which likely contributed to enhancement of ICB efficacy in controlling NSCLC tumor growth. Given the need for synergistic combinations with ICBs, shIDO-ST treatment is an attractive approach that could augment response rates in NSCLC patients by reprogramming intratumoral suppressive immune subsets to a more immunostimulatory state.

\section{YIA20-005: Rapid Point of Care assay by isothermal PCR for Acute Promyelocytic Leukemia Diagnosis Cecilia Yeung, $\mathrm{MD}^{\mathrm{a}, \mathrm{b}}$; Bret Helton, $\mathrm{BS}^{\mathrm{a}}$; and Jerald Radich, MD ${ }^{\mathrm{a}, \mathrm{b}}$ aFred Hutchinson Cancer Research Center, Seattle WA; bUniversity of Washington, Seattle WA}

Background: Acute promyelocytic leukemia (APL) accounts for $\sim 10 \%$ of acute myelogenous leukemia (AML) and may present as a medical emergency with risk of early death. Since the introduction of induction therapy with all-trans retinoic acid and arsenic, APL has become both the most curable yet deadliest form of AML. Definitive diagnoses are often not possible during a STAT evaluation because of APL's unique morphological features that lack immature blasts and instead are either promyelocytes, or in another variant (the microgranular variant) appear like monocytes. Further complicating the picture is the inability of definitive identification by flow cytometry, which relies on the lack of CD34 and other typical blast markers seen in AML. Therefore, we propose a rapid point of care diagnostic assay is needed. Methods: We developed an iPCR reaction targeting PML-RARA with Recombinase Polymerase Amplification (RPA). We were able to use the products from the RPA reaction and apply to a lateral flow device for visualization of a positive PML-RARA fusion. Samples included cell lines, NB4 with known PML-RARA fusion, K562 without any RARA fusion and 16 patient samples including 12 APL, 4 CML. Patient samples were processed within 24-48 hours of draw from fresh blood or bone marrow and RNA isolated via trizol per manufacture protocol. Target input RNA was 500ng/ul, however some patient samples had low RNA yield and suboptimal input volumes were used. Results: We tested 59 samples on known cell lines and determined that our RPA reactions have a sensitivity of $100 \%$ (CI: $92.45 \%$ to $100.00 \%$ ), a specificity of $100 \%$ (CI: $73.54 \%$ to $100 \%$ ) of $100 \mathrm{ng} / \mathrm{uL}$, a $\mathrm{PPV} / \mathrm{NPV}$ of $100 \%$ and $100 \%$ respectively, and accuracy is
$100 \%$ (CI: $93.94 \%$ to $100.00 \%$ ). Our APL RPA reaction could be functional down to 30 degrees reaction which is optimal for a POC design. Limit of detection (LOD) can now detect down to 5ng of RNA from NB4 cell lines diluted with K562 RNA (for a total input of 500ng). On patient samples $7 / 12$ were shown to be positive, the 5 negative samples had low input volumes (optimal $=500 \mathrm{ng}$ ) below the LOD. Conclusions: Our initial design for this RPA assay has been able to reliably detect the PML-RARA fusion RNA transcript in a $30^{\circ}$ reaction without the need of any additional electronic equipment. The potential for a point of care device is feasible, however, more optimization for improved sensitivity, lower input RNA requirements, and to achieve a lower limit of detection is needed.

\section{YIA20-006: Preclinical Pediatric MATCH}

Elizabeth Stewart, MD; Kaley Blankenship, BS; Lauren Hoffmann, BS; and Burgess Freeman, $\mathrm{PhD}$

St. Jude Children's Research Hospital, Memphis, TN

Background: The overall survival rate for children with solid tumors has plateaued over the past 2 decades and survival rates remain at or below $30 \%$ for children with recurrent or metastatic disease. This plateau comes at a time when pediatric oncologists have access to an unprecedented diversity and number of new oncology drugs. In addition to these advances in drug development, it is now feasible to obtain complete genomic profiling of each patient's tumor using Next Generation Sequencing (NGS). Several clinical genomic efforts have been launched including the Pediatric MATCH trial that seeks to evaluate molecularly targeted therapies in children with advanced cancers. As the concept of a "druggable" mutation is one that is still being refined in pediatric solid tumors, this study seeks to use a diverse collection of orthotopic murine patient derived xenografts (PDXs) generated through the Childhood Solid Tumor Network (CSTN) to study these potentially actionable mutations in the preclinical setting. Methods: Over 60 PDX models representing the major pediatric solid malignancies in the CSTN were analyzed using histology, electron microscopy, gene expression profiling, whole genome sequencing, exome sequencing, RNA-seq and DNA methylation. Pharmacokinetic studies of the drugs being used in the Pediatric MATCH trial were performed in tumor bearing mice to determine matched human AUC-guided dosing. Preclinical studies were performed using PDX models with identified druggable mutations according to the Pediatric MATCH study as well as those lacking the mutation for control. Results: Several druggable mutations were identified in the genomic analysis of the PDX tumors including ALK, ROS1, TSC1, FGFR4, MAPK, SMARCB1, ATM, and NTRK1. Mouse equivalent dosing was successfully obtained through PK studies for all of the drugs that match the found mutations. Preclinical efficacy 
studies using the PDX tumors with and without identified druggable mutations are currently underway. Results of these studies will be presented at the annual meeting. Conclusions: The testing of molecularly targeted therapeutics in PDX models of pediatric solid tumors is feasible and may be beneficial to understanding the driver mutations and underlying biology of these malignancies. We hope to continue to use these PDX models created through the CSTN to help prioritize agents for further clinical trial development for pediatric patients.

\section{BEST PRACTICES IN IMPLEMENTATION AND USE OF CLINICAL PRACTICE GUIDELINES}

\begin{abstract}
BPI20-017: Innovating Patient-Facing Care Pathways in Breast Cancer Using the 4R model, 4R = Right Information / Care / Patient / Time

Della F. Makower, MDa; Christine Brezina Weldon, MBA;

Julia R. Trosman, PhD, MBA ${ }^{\text {b; }}$ Kent Hoskins, MD, BA;

Cathleen Schaeffer, RN, BSN, OCN, CBCNc; Bruce D. Rapkin, PhDa; Melissa A. Simon, MD, MPH' ; Al B. Benson, MD, FACP, FASCOd; and William J. Gradishar, MD, FASCO FACPd

${ }^{a}$ Albert Einstein College of Medicine, Bronx, NY; ${ }^{b}$ Northwestern University, Feinberg School of Medicine, Chicago, IL; 'University of Illinois Cancer Center, Chicago, IL; ${ }^{d}$ Robert H. Lurie Comprehensive Cancer Center of Northwestern University, Chicago, IL
\end{abstract}

Background: ASCO's “Criteria for High-Quality Clinical Pathways in Oncology" calls for development of multimodality patient focused pathways. However, clinical pathways are typically created for provider use. The innovative $4 \mathrm{R}$ model (right information and right care for the right patient at the right time), developed by the Center for Business Models in Healthcare and Northwestern University, is an approach to pathways which empower patients in conjunction with their care team. The $4 \mathrm{R}$ model incorporates recommendations from the Institute of Medicine, Medicare's OCM, and National Comprehensive Cancer Network's guidelines: multimodality planning from diagnosis to survivorship or hospice, specifying clinical team and their responsibilities; and inclusion of definitive and supportive cancer care in the plan. This project was funded by an

BPI20-017 Table: Patient Self-Management

\begin{tabular}{|lccc|}
\hline & $\begin{array}{c}\text { 4R Cohort } \\
(\mathbf{n = 5 0 )}\end{array}$ & $\begin{array}{c}\text { Historical cohort } \\
(\mathbf{n}=\mathbf{1 2 3})\end{array}$ & P-value \\
\hline Knowing stage of your cancer & $92 \%$ & $72 \%$ & 0.0043 \\
\hline $\begin{array}{l}\text { How well care plan was } \\
\text { explained }\end{array}$ & $90 \%$ & $63 \%$ & 0.0002 \\
$\begin{array}{l}\text { Never or seldom felt not in } \\
\text { control of my care }\end{array}$ & $74 \%$ & $54 \%$ & 0.0166 \\
$\begin{array}{l}\text { Able to manage your care } \\
\text { well or very well }\end{array}$ & $86 \%$ & $72 \%$ & 0.0512 \\
\hline \begin{tabular}{l} 
Care plan clear or very clear \\
\hline
\end{tabular} & $84 \%$ & $78 \%$ & 0.413 \\
\hline
\end{tabular}

NCCN/Pfizer grant. Overall Goal: To help breast cancer patients, their families/caregivers, and their care team manage guideline indicated care through use of $4 \mathrm{R}$ patient care sequences / pathway plans. Methods: This project implemented the $4 \mathrm{R}$ model for a target population of breast cancer patients and care teams at two major institutions serving predominantly minority and underserved patients. Patient self-management metrics were collected via patient survey prior to (historical) and after implementation (4R cohort). Descriptive statistics and Fisher's exact test were used for analysis Results: Patients who received the care sequence plan reported that their care was explained well/very well $90 \%(45 / 50)$ as compared to $63 \%(77 / 123)$ of patients who did not receive care sequence plans, $\mathrm{p}=0.0002$. There were statistically significant improvements in patients "knowing stage of your cancer", $p=0.0043$; and in "never or seldom felt not in control of care", $p=0.0166$. Of patients who received care sequence plans, $90 \%(45 / 50)$ found plan very useful or useful. Conclusion: Our project showed that the $4 \mathrm{R}$ model is a promising patient selfmanagement method providing early breast cancer patients with their own care pathways. The patient care sequence plans markedly improved several aspects of patient self-management as well as delivery of multimodality care. An ongoing 4R collaboration continues development of the $4 \mathrm{R}$ model to address remaining gaps and improve $4 \mathrm{R}$ effectiveness.

\section{CLINICAL ONCOLOGY}

CLO20-030: Sex-Based Disparities in Receipt of Care and Survival in Malignant Pleural Mesothelioma

Andrew R. Barsky, MDa; Christopher A. Ahern, $\mathrm{PhD}^{\mathrm{b}}$;

Sriram Venigalla, MDa; Vivek Verma, MDc; Emily J. Anstadt, MD, PhDa Christopher M. Wright, MDa; Ethan B. Ludmir, MD';

Christopher G. Berlind, $\mathrm{PhD}^{\mathrm{b}}$; William D. Lindsay, $\mathrm{MS}^{\mathrm{b}}$;

Surbhi Grover, MD, MPHa; Keith A. Cengel, MD, PhDa;

and Charles B. Simone, II, MDe

aPerelman School of Medicine, Department of Radiation Oncology University of Pennsylvania, Philadelphia, PA; ${ }^{b}$ Oncora Medical, Philadelphia, PA; 'Allegheny General Hospital, Pittsburgh, PA; ¿University of Texas M.D. Anderson Cancer Center, Houston, TX; eNew York Proton Center, New York, NY

Purpose: Despite accounting for a minority of malignant pleural mesothelioma (MPM) diagnoses, females may experience differential survival relative to males. It is currently unclear if differences in receipt of treatment between sexes contribute to this survival disparity. We thus utilized the National Cancer Database (NCDB) to assess patterns-of-care and overall survival (OS) in male vs. female patients with MPM. Methods: Patients with histologically-confirmed MPM treated from 2004-2013 were identified from the NCDB. The association between female sex and OS was assessed using multivariable (MV) 
Cox proportional hazards models with propensity score (PS) matching. Patterns-of-care were assessed using MV logistic regression. The overall treatment effect was tested in subsets of patients by treatment strategy, histology, and clinical stage. Results: A total of 18,799 patients were identified, of whom 14,728 (78\%) were male, and 4,071 (22\%) were female. Females were more likely to present at a younger age, with less comorbidity, and with epithelioid histology (all $\mathrm{p} \leq 0.001$ ). Despite these favorable prognosticators, females were less likely to receive surgery or chemotherapy compared to males (both $\mathrm{p} \leq 0.001$ ). On MV analysis, female sex was associated with improved OS (hazard ratio (HR) 0.83 , 95\% confidence interval (CI) $0.80-0.86, \mathrm{p} \leq 0.001$ ), and this association remained significant following PS-matched MV survival analysis (HR 0.85, 95\% CI 0.80-0.89, $\mathrm{p} \leq 0.001$ ). This survival disparity was noted across all stages but only in patients with epithelioid histology $(p \leq 0.001)$. Conclusions: In the largest such analysis to date using a nationally representative contemporary cohort of over 18,000 patients with MPM, we demonstrate that surgery and chemotherapy are disproportionately underutilized in female patients with MPM. Despite this concerning disparity, female sex is independently associated with improved OS relative to males, even when adjusting for potential confounders. The decreased hazard of death associated with female sex appears most pronounced in patients with epithelioid histology, and such an association is more limited or even non-existent in non-epithelioid histologies. As females experience improved OS compared to males with MPM, efforts should be made to reduce disparities in the delivery of potentially life-extending treatments to this population. Further research to understand factors that lead to sex disparities in MPM is warranted.

\section{CLO20-034: First U.S. Prospective Evaluation of Performance of 64Cu DOTATATE PET/CT in Somatostatin Expressing Neuroendocrine Tumors \\ Ebrahim S. Delpassand, MDa; Rodolfo Nunez, MDa; D. Ranganathan, $\mathrm{PhD}^{\mathrm{b}} ; \mathrm{N}$. Wagh, $\mathrm{PhD}^{\mathrm{b}}$; A. Shafie, $\mathrm{MD}^{\mathrm{a}}$; A. Gaber, MDa; I. Tworowska, PhD ${ }^{\mathrm{b}}$; and A. Abbasi, MD ${ }^{\mathrm{b}}$ ${ }^{a}$ Excel Diagnostics and Nuclear Oncology Center, Houston, TX; ${ }^{b}$ RadioMedix, Houston, TX}

Introduction: 68 Ga-labeled Somatostatin (SST) analogues are highly sensitive and specific PET radiopharmaceuticals for imaging SST expressing neuroendocrine tumors (NET). However, their major shortcomings are short half-life of 68 minutes, dependency on Ga-68 generator, and hence availability only in limited geographical regions. Cu-64 DOTATATE addresses all these shortcomings with half-life of 12.6 hours, cyclotron production, centralized high scale manufacturing and distribution throughout the country. Objective: The study aim was to prospectively evaluate the safety and diagnostic performance of $64 \mathrm{Cu}$-DOTATATE PET/CT in detection of SST expressing NET. Method: All subjects received $4.0 \pm 10 \% \mathrm{mCi}$ of $64 \mathrm{Cu}$-DOTATATE and $\mathrm{PET} / \mathrm{CT}$ imaging was performed $60 \pm 15$ minutes after injection. PET/CT images were read by three independent nuclear medicine physicians blinded to clinical information. Separately, Independent oncologist determined disease or no disease as well as local or metastatic status

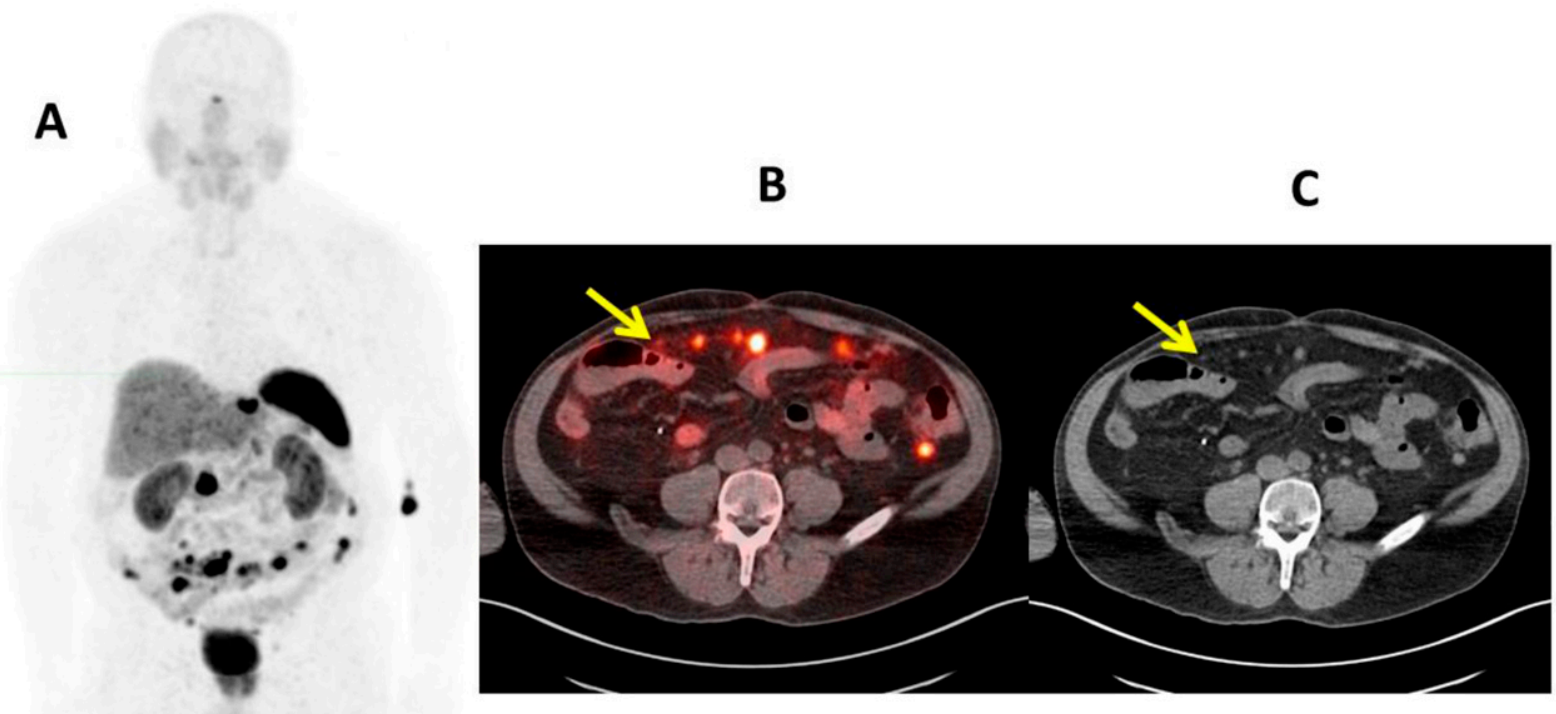

CL020-034 Figure. Maximum intensity projection image (A) of a patient with metastatic small bowel carcinoid tumor. Small (yellow arrow points to a 3 mm lesion) omental and peritoneal tumor implants are seen on the PET/CT fused image (B), and the corresponding CT image (C). 
of the subject, from all available Standard of Truth (SOT) pathology, clinical, and conventional imaging studies. A total of 63 subjects were considered for statistical analysis. Results: All the images were of high quality. Reader 1 had a sensitivity and specificity of $91 \%$ and $97 \%$; reader 2 of $91 \%$ and $80 \%$, and reader 3 of $91 \%$ and $90 \%$, respectively. It is important to note that failures in detecting disease were retrospectively reviewed by the investigators after the database was locked. Upon review of these failures it was noted that three SOT image reads determined to be positive for disease by the oncologist, were in fact negative for disease. Each of these subjects had their primary tumor resected prior and anatomical imaging confirming no evidence of disease prior to determining the SOT and as such should have had a SOT determined as "no disease". Taking this into account, for readers $1 \& 3$ and the majority read would have had a sensitivity of $100 \%$, specificity of $96.8 \%$, PPV of $96.7 \%$, NPV of $100 \%$, and accuracy of $98.4 \%$. Reader 2 would have had a sensitivity of $100 \%$, specificity of $81.8 \%$, PPV of $83.3 \%$, NPV of $100 \%$, and accuracy of $90.5 \%$. All patients tolerated $64 \mathrm{Cu}$-DOTATATE well, with no serious adverse reactions. Conclusion: $64 \mathrm{Cu}$-DOTATATE PET/CT is a safe imaging technique that provides high quality images (figure) and excellent accuracy (98\%) for detection of SST expressing NETs.

\section{CLO20-039: Patient (pt)-Reported Outcomes (PRO) in Patients With HER2-Negative Locally Advanced/ Metastatic Breast Cancer (LA/mBC) and a Germline BRCA1/2 Mutation (gBRCA1/2 mut) Receiving Talazoparib vs. Physician's Choice of Chemotherapy (PCT): A Focus on EMBRACA Racial Subgroups \\ Sara A. Hurvitz, MDa; Ruben G. W. Quek, PhD'; \\ Helen Bhattacharyya, $\mathrm{PhD}^{\mathrm{b}}$; Johannes Ettl, MDc; \\ Anthony Gonçalves, $\mathrm{MD}, \mathrm{PhD}^{\mathrm{d}}$; and Hope S. Rugo, $\mathrm{MD}^{\mathrm{e}}$ \\ aUniversity of California Los Angeles, Los Angeles, CA; 'bPfizer, Inc.,

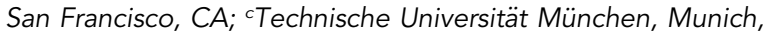 Germany; ${ }^{d}$ Aix-Marseille Université, Marseille, France; e UCSF Helen Diller Family Comprehensive Cancer Center, San Francisco, CA}

Background: Key subgroup analyses of EMBRACA (NCT01945775) demonstrated improvements in progression-free survival for talazoparib vs PCT in white and non-white pts with HER2-negative g BRCA1/2 mut LA/ $\mathrm{mBC}$. We present post hoc analyses evaluating PRO in these racial subgroups. Methods: PRO was assessed at baseline, start of treatment cycle (every 3 wks), and end of treatment using the European Organization for Research and Treatment of Cancer Quality of Life Questionnaire Core 30 (EORTC QLQ-C30) and its breast cancer module, QLQ-BR23. Higher scores indicate better functioning/global health status (GHS)/quality of life (QoL) or worse symptom severity. PRO analyses, performed separately in white/non-white pts for GHS/QoL functional and symptom scales, included comparison of overall change from baseline (per longitudinal repeated measures mixed-effects model) and time to definitive clinically meaningful deterioration (TTD; per survival analysis methods) using a Cox proportional hazards model and stratified log-rank test. Results: Baseline PRO scores in the evaluable population were similar between the talazoparib (white/non-white, $n=173 / 89$ ) and PCT (white/non-white, $\mathrm{n}=84 / 30$ ) arms. A statistically significant estimated overall change from baseline in GHS/ QoL favored talazoparib vs PCT for white (7.4 [95\% confidence interval $\{\mathrm{CI}\}: 2.6,12.2] ; \mathrm{P}=0.003$ ) and nonwhite (10.1 [95\%CI: $3.7,16.5] ; \mathrm{P}=0.002)$ pts. A statistically significant estimated overall change from baseline in $\mathrm{pt}$ reported pain symptoms favored talazoparib vs PCT for white ( $\mathrm{P}<0.001)$ and non-white $(\mathrm{P}=0.02)$ pts. A statistically significant delay in TTD favoring talazoparib vs PCT was observed in GHS/QoL for both white (median [m]: 21.1 vs 6.3 mos, hazard ratio $[\mathrm{HR}]=0.38$ [95\% CI: $0.24,0.59]$; P $<0.001$ ]) and non-white (m: [not reached] vs $10.4 \mathrm{mos}$, $\mathrm{HR}=0.38$ [95\% CI: 0.17, 0.83]; $\mathrm{P}=0.01$ ) pts. A delay in TTD favoring talazoparib was observed in pain symptoms for both white (m: 22.7 vs $10.3 \mathrm{mos}, \mathrm{HR}=0.29, \mathrm{P}<0.001)$ and non-white (m: 23.0 vs 7.5 mos, $\mathrm{HR}=0.63, \mathrm{P}=0.22$ ) pts. Conclusions: In pts with HER2-negative g BRCAl/2 mut $\mathrm{LA} / \mathrm{mBC}$, talozaparib vs PCT resulted in significantly better change from baseline and delayed TTD in GHS/QoL and pt reported pain symptoms in white pts; similar results were observed in non-white pts. Future real-world PRO studies across different racial subgroups are warranted.

\section{CLO20-055: Optimal Timing of Radical Surgery From Diagnosis in Muscle Invasive Bladder Cancer (MIBC) \\ Saurabh Parasramka, MD; Quan Chen, DrPH; Bin Huang, DrPH., MS; Peng Wang, MD, PhD; and Zin Myint, MD \\ University of Kentucky, Lexington, $K Y$}

Background: Non-metastatic muscle invasive bladder cancer (MIBC) is treated with radical cystectomy and survival is closely associated with final pathologic staging. For patients undergoing primary surgery there is evidence that delay $>90$ days after diagnosis adversely effects survival outcomes. Neoadjuvant chemotherapy (NAC) has become the standard of care for appropriate patients, which delays definitive surgery. Optimal timing of surgery from the time of diagnosis is uncertain when done after NAC. We studied this question using National Cancer Database (2004-2015). Methods: We identified patients > 18 years with MIBC (cT2-T4aN0M0 \& cT1T4aN1M0). All patient's received NAC within 6 months of diagnosis and underwent surgery within 9 months of the start of NAC. We excluded patients who died within 30 days of surgery. Time from diagnosis to surgery was stratified into five cohorts; <18, 19-22, 23-26, 27-30, and $>31$ weeks. Descriptive analysis, Kaplan-Meier plots, Log-Rank tests for univariate and proportional hazards models for multivariate survival analyses were performed. 
Results: 4168 patients were identified; $75 \%$ were males, $70 \%$ cases were stage 2 , and $73 \%$ had charlson-deyo score (CS) of 0 . Median time to surgery from diagnosis was 154 days. Majority (60\%) were treated at academic and $24 \%$ at comprehensive community. Only $28 \%$ achieved complete pathological complete response rate (Tis or T0). On univariate analysis patients receiving surgery within 30 weeks of diagnosis had significantly better survival $(\mathrm{p}<0.05)$. Receiving chemotherapy within 3 months of diagnosis was significantly associated with survival benefit on univariate analysis $(\mathrm{p}<0.05)$ which was not present on multivariate analysis HR 0.99 (0.82-1.20). In addition patients with CS of ' 1 ' and age $>75$ had significantly worse survival with HR 1.15 (1.02-1.28) and 1.63(1.28-2.09). Achieving less than pathological complete response defined as (pTis, pT0, pTa) was associated with significantly poor survival $(\mathrm{p}<0.0001)$ Conclusions: Our study shows that appropriate patients with MIBC benefit from receiving surgery within 30 weeks of diagnosis.

CLO20-059: Comparison of a Single Dose Immediate Instillation of Mitomycin C $20 \mathrm{mg}$ Versus $40 \mathrm{mg}$ in Prevention of Tumour Recurrence After Resection of Non Muscle Invasive Bladder Cancer

Shree Vishnu Siddarth, MS, MCh; Ginil Kumar Pooleri, MS, MCh; and Georgie Mathew, MS, MCh

Amrita institute of Medical Sciences and Research, Kochi, Kerala, India

Introduction: The recurrence following a transurethral resection (TUR) of non-muscle invasive bladder cancer
(NMIBC) remains relatively high. A single immediate postoperative instillation of mitomycin $C$ (MMC) reduces the rate of recurrence in the first two years but does not improve the progression of tumour or overall survival. The European Association of Urology (EAU) and American Urological Association (AUA) guidelines recommend a single immediate postoperative instillation of chemotherapeutic agent but the dosing and concentration of mitomycin $\mathrm{C}$ to be instilled is not clear. There are very limited randomized control trials (RCT) comparing the different dosing and concentration regimens of MMC in prevention of recurrence of NMIBC following a TUR. Materials and Methods: A randomized, prospective, two-arm, open-label, single centre, pilot study was conducted between April 2017 and April 2018 was conducted at a tertiary care centre in South India. Patients were randomized into MMC 20mg group ( $\mathrm{n}=$ $34)$ or MMC $40 \mathrm{mg}$ group $(\mathrm{n}=35)$ and analysed following a TUR for NMIBC. Results: At the end of 12 months, recurrence free rates (RFR) for MMC 20mg and MMC $40 \mathrm{mg}$ were $57.1 \%$ and $68.6 \%$ respectively. The mean recurrence free interval (RFI) for MMC 20mg and MMC 40mg group was 11.1 months and 10.8 months respectively. The difference in RFR and RFI between the two groups was not statistically significant. Conclusion: A single immediate postoperative instillation of $20 \mathrm{mg}$ mitomycin $\mathrm{C}$ is not inferior and is comparable to $40 \mathrm{mg}$ in preventing tumour recurrence in NMIBC.

\begin{tabular}{|c|c|c|c|c|c|c|}
\hline \multirow[b]{2}{*}{ Risk stratification } & \multirow[b]{2}{*}{ Randomisation group } & \multicolumn{4}{|c|}{ Recurrence free rate (\%) } & \multirow[b]{2}{*}{$p$ value (log rank test) } \\
\hline & & At 3 months & At 6 months & At 9 months & At 12 months & \\
\hline \multirow[t]{2}{*}{ Overall patients } & MMC $20 \mathrm{mg}$ & 100 & 91.4 & 82.9 & 57.1 & \multirow[t]{2}{*}{0.412} \\
\hline & MMC 40mg & 94.3 & 82.9 & 82.9 & 68.6 & \\
\hline \multirow[t]{2}{*}{ Low risk patients } & MMC $20 \mathrm{mg}$ & 100 & 86.7 & 86.7 & 60 & \multirow[t]{2}{*}{0.386} \\
\hline & $\mathrm{MMC} 40 \mathrm{mg}$ & 100 & 84.6 & 84.6 & 76.9 & \\
\hline \multirow[t]{2}{*}{ Intermediate risk patients } & MMC $20 \mathrm{mg}$ & 100 & 100 & 75 & 50 & \multirow[t]{2}{*}{0.476} \\
\hline & MMC $40 \mathrm{mg}$ & 60 & 40 & 40 & 40 & \\
\hline \multirow[t]{2}{*}{ High risk patients } & MMC $20 \mathrm{mg}$ & 100 & 93.3 & 80 & 53.3 & \multirow[t]{2}{*}{0.256} \\
\hline & MMC $40 \mathrm{mg}$ & 100 & 94.1 & 94.1 & 70.6 & \\
\hline \multirow[t]{2}{*}{ Ta stage } & MMC $20 \mathrm{mg}$ & 100 & 89.5 & 84.2 & 57.9 & \multirow[t]{2}{*}{0.618} \\
\hline & MMC $40 \mathrm{mg}$ & 90 & 75 & 75 & 70 & \\
\hline \multirow[t]{2}{*}{ T1 stage } & MMC $20 \mathrm{mg}$ & 100 & 93.3 & 80 & 53.3 & \multirow[t]{2}{*}{0.376} \\
\hline & MMC $40 \mathrm{mg}$ & 100 & 93.3 & 93.3 & 66.7 & \\
\hline \multirow[t]{2}{*}{ Low grade } & MMC $20 \mathrm{mg}$ & 100 & 89.3 & 82.1 & 53.6 & \multirow[t]{2}{*}{0.450} \\
\hline & MMC 40mg & 92.6 & 81.5 & 81.5 & 66.7 & \\
\hline \multirow[t]{2}{*}{ High grade } & MMC $20 \mathrm{mg}$ & 100 & 100 & 83.3 & 66.7 & \multirow[t]{2}{*}{0.732} \\
\hline & MMC 40mg & 100 & 87.5 & 87.5 & 75 & \\
\hline
\end{tabular}


CLO20-062: Comparative Efficacy of Elotozumab With Panobinostat and Ixazomib in Multiple Myeloma; A Meta-Analysis

Nishanth Thalambedu, MDa; Waqas Ullah, MDa; Ammar Ashfaq, MDa; Yasir Khan, MDa; Ammar Ashfaq, MDa; Qian Zhang, MDa; and Mishal Shaukat, MD ${ }^{\mathrm{b}}$

${ }^{a}$ Abington Jefferson Health, Abington, PA; ${ }^{b}$ Combined military hospital, Lahore, Pakistan

Background: Multiple Myeloma(MM) has a variable response rate to different chemotherapies. We sought to determine Elotuzumab efficacy by assessing 50\%, 90\%, and $100 \%$ decrease in the $M$ protein levels termed as partial response (PR), very good partial response (VGPR), and complete response (CR) respectively, in MM patients. Methods: A comprehensive literature search on MedLine and Cochrane databases identified five relevant clinical trials (984 patients). The response rate was analyzed on a fixed-effect model using an unadjusted odds ratio (OR) on RevMan 5.3. Results: Elotuzumab was associated with a net

CLO20-062 Figure 1: Odds ratio of VGPR rates between Elotuzumab and Other multiple myeloma medications.

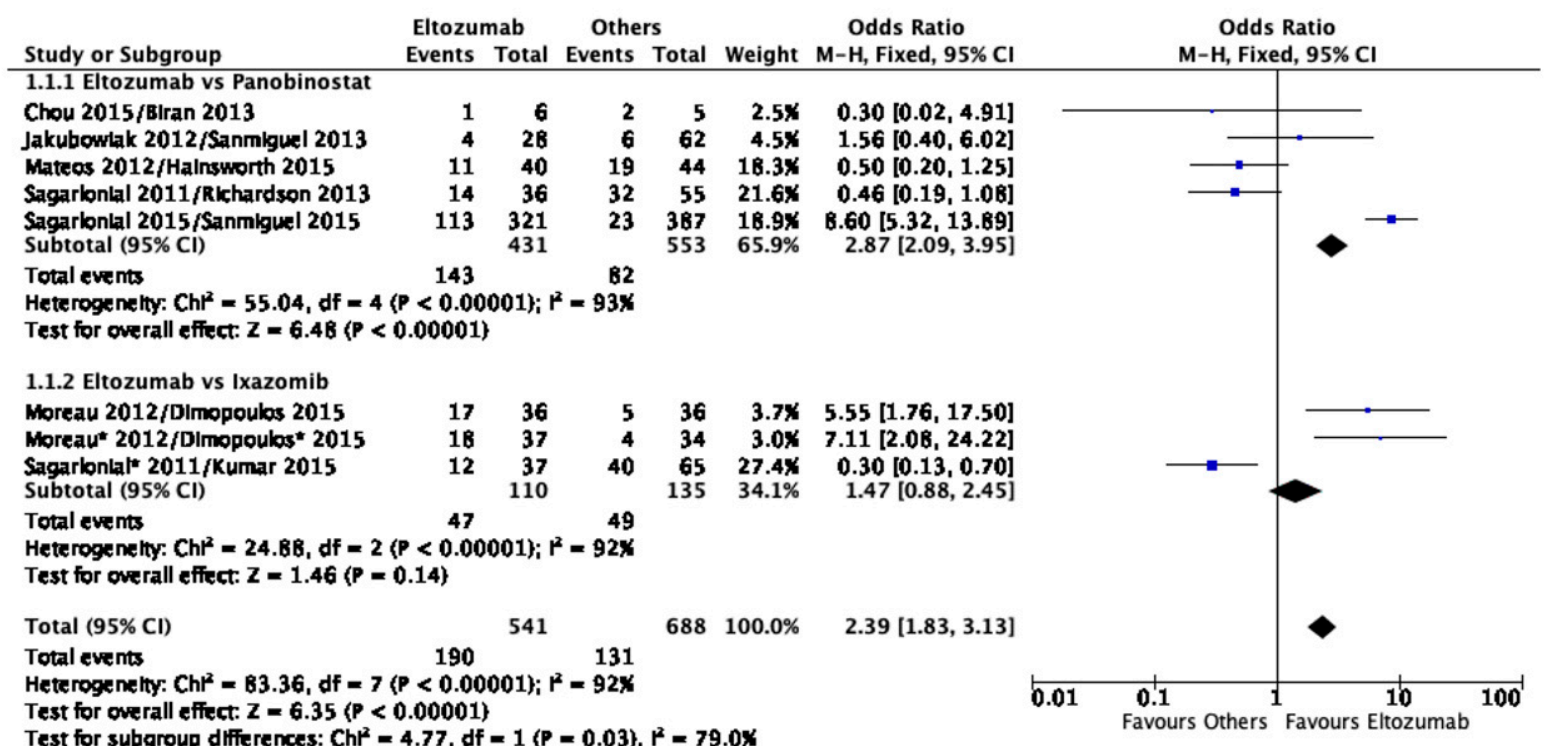

CLO20-062 Figure 2: Odds ratio of PR rates between Elotuzumab and Other multiple myeloma medications.

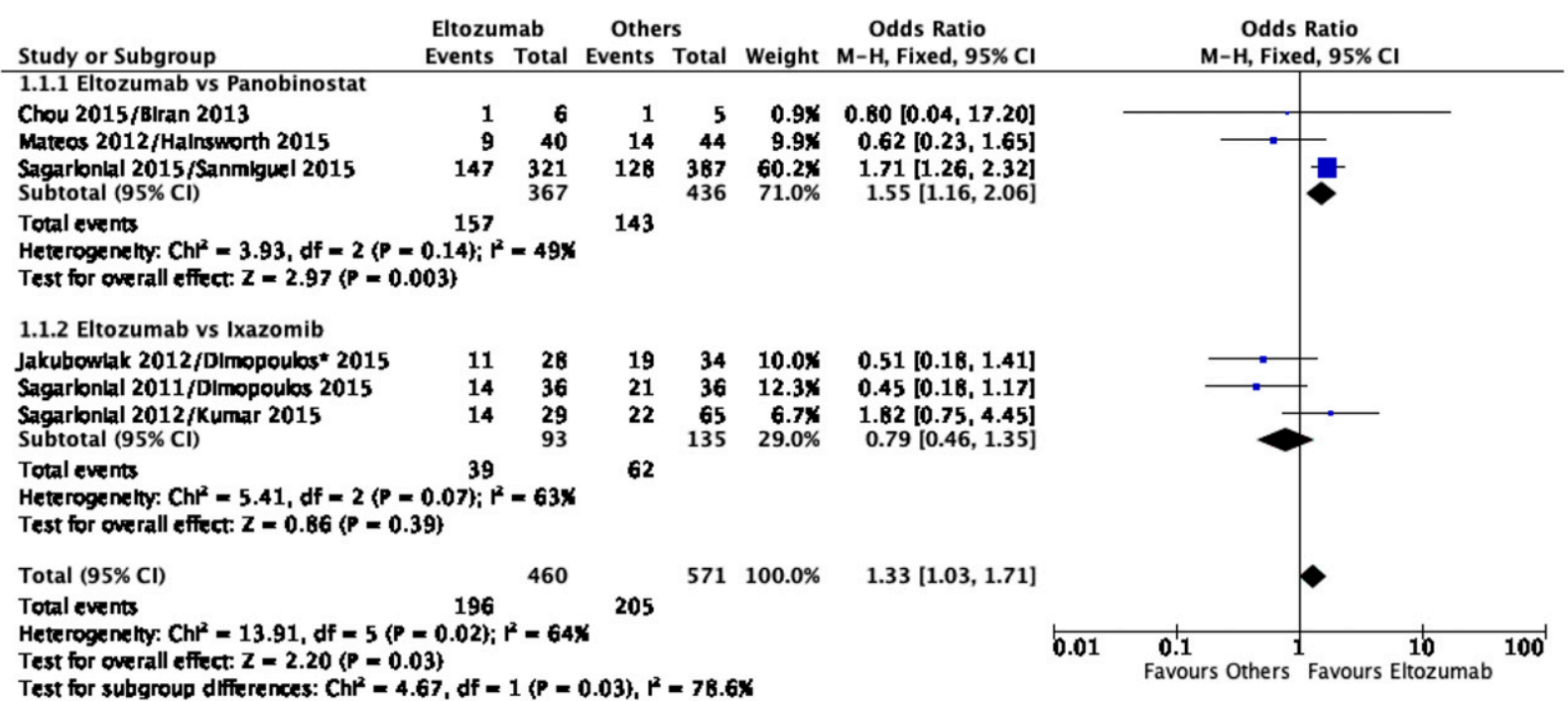


$58 \%$ higher odds to achieve VGPR against panobinostat and Ixazomib combined (OR 2.39, 95\% CI 1.83-3.13, $\mathrm{p}<0.01$ ). (Figure 1) Compared to panobinostat, Elotozumab had a $66 \%$ higher chance to achieve VGPR (OR 2.87, 95\% CI 2.09-3.95, $\mathrm{p}<0.01$ ). Subgroup analysis of three studies comprising of 245 patients showed that Elotozumab had a $32 \%$ higher but non-significant VGPR rate compared to Ixazomib alone (OR 1.47, 95\% CI 0.88-2.45, $\mathrm{p}=0.14)$. Elotuzumab was also shown to achieve significantly higher rates of partial response (PR) (OR 1.33, 95\% CI 1.03-1.71, $\mathrm{p}=0.03$ ) compared to other medications. (Figure 2) However, the complete response rates were significantly lower in Elotuzumab compared to other medications (OR 0.29 , 95\% CI 0.19$0.45, \mathrm{p}=<0.00001$ ). (Figure 3 , available online, in this abstract, at JNCCN.org) Conclusion: Elotuzumab is associated with a substantially higher rate of achieving VGPR and PR rates. However, the complete response rate was $70 \%$ lower compared to Panobinostat and Ixazomib.

\section{OUTCOMES AND HEALTH SERVICES RESEARCH}

HSR20-076: Provider Versus Patient Reported Outcomes (PROs) in Colorectal Cancer (CRC) Patients (pts)

Undergoing Systemic Therapy (Ctx): A Real

World Experience

Sonali Agrawal, BS ; Caitlin R. Meeker, MPH

Sandeep Aggarwal, MD'; Elizabeth A. Handorf, PhDa;

Sunil Adige, MDa; Efrat Dotan, MDa; Crystal S. Denlinger, MDa;

William H. Ward, MDa; Jeffrey M. Farma, MD ${ }^{a}$

and Namrata Vijayvergia, $\mathrm{MD}^{\mathrm{a}}$

${ }^{a}$ Fox Chase Cancer Center, Philadelphia PA; ${ }^{b}$ Drexel University School of Medicine, Philadelphia PA

Background: There has been increasing interest in the integration of PROs as part of routine toxicity (tox) assessment and reporting as this may improve tolerance and survival on systemic therapy. Our study aimed to highlight the differential reporting of tox by CRC pts and their providers during Ctx with a focus on actionable tox.
We hypothesize that for such tox, under-reporting by providers will be observed less frequently. Methods: With IRB approval, $40 \mathrm{CRC}$ pts undergoing Ctx for CRC at a National Cancer Institute-designated comprehensive cancer center were prospectively enrolled to this observational study. Pts completed PRO-CTCAE forms at the start of therapy and at the subsequent follow up visit. Severity of tox was ranked on a 5-point scale ("none", "mild", "moderate", "severe", "very severe"). Provider-assessed tox were collected via questionnaires administered in real time (for grade $3+$ tox) and also pt chart review. Actionable tox were defined as those that commonly may limit Ctx or require a dose modification (mouth sores, diarrhea, nausea, vomiting, rash, hand-foot syndrome, and neuropathy). Exact McNemar's test was used to analyze agreement in reporting between patient and provider for each tox. Results: Of 40 pts enrolled, $75 \%$ had colon cancer, $70 \%$ were Stage IV, and $63 \%$ had an ECOG performance status of 0 . The median age at enrollment was 55 years and the cohort included 55\% male, $80 \%$ white, and 58\% married adults. Most (93\%) pts were treated with chemotherapy (others were on clinical trials with novel agents). Most common grade $3+$ tox reported by providers included nausea (10\%) and neuropathy $(8 \%)$, while the common clinically significant tox reported by pts were neuropathy $(20 \%)$, fatigue $(20 \%)$, and anxiety (15\%). Reported tox were compared to assess degree of agreement in reporting between pts and providers and significant differences were found (Table). Conclusion: Our study provides real world evidence that physicians consistently under-report and document tox from Ctx compared to pts with CRC. This gap did not narrow for actionable tox either. Incorporating PROs in tox reporting and management may provide additional information to physicians to help tailor Ctx and toxicity management plans for an individual pt. Ongoing analysis to correlate tox data with 2-year survival will provide further information about the clinical utility of PROs in ongoing cancer care.

HSR20-076 Table: Provider versus patient reported outcomes

\begin{tabular}{|lrrrr|}
\hline Toxicity (any grade) & Provider Only & Patient Only & Both Provider \& Patient & Exact McNemar Test (P value) \\
\hline Mouth sores* & $2.5 \%$ & $22.5 \%$ & $2.5 \%$ & $\mathbf{0 . 0 2 1}$ \\
\hline Increased flatulence & $0 \%$ & $42.5 \%$ & $5 \%$ & $\mathbf{1 . 5 2 6 e - 0 5}$ \\
\hline Diarrhea* & $5 \%$ & $45 \%$ & $17.5 \%$ & $\mathbf{0 . 0 0 0 4 0 2 5}$ \\
\hline Shortness of breath & $2.5 \%$ & $30 \%$ & $7.5 \%$ & $\mathbf{0 . 0 0 3 4}$ \\
\hline Hair loss & $0 \%$ & $22.5 \%$ & $2.5 \%$ & $\mathbf{0 . 0 0 3 9}$ \\
\hline Neuropathy & $2.5 \%$ & $37.5 \%$ & $27.5 \%$ & $\mathbf{0 . 0 0 0 5 1 8 8}$ \\
\hline Fatigue & $5 \%$ & $52.5 \%$ & $35 \%$ & $\mathbf{6 . 6 0 4 e - 0 5}$ \\
\hline Anxiety & $2.5 \%$ & $42.5 \%$ & $12.5 \%$ & $\mathbf{0 . 0 0 0 1 4 5}$ \\
\hline
\end{tabular}

${ }^{\star}$ An actionable toxicity 
HSR20-085: Real-World Study of Factors Associated With Breast Conserving Surgery for Females Diagnosed With Early Stage Breast Cancer

Judy George, PhDa; Joseph Tkacz, MSa;

M. Christopher Roebuck, PhD, MBA ${ }^{\text {b; }}$ Fredy Reyes, $\mathrm{PhD}^{\mathrm{a}}$;

Yull E. Arriaga, MDa; Gretchen P. Jackson, MD, PhD;

and Irene Dankwa-Mullan, MD, MPH ${ }^{\mathrm{a}}$

alBM Watson Health, Cambridge, MA; ${ }^{b} R X$ Economics, LLC, Hunt Valley, MD; ' Vanderbilt University Medical Center, Nashville, TN

Background: Breast Conserving Surgery (BCS) is now considered an acceptable alternative to mastectomy for patients with early stage invasive breast cancer (ESBCa). Limited research examining factors influencing choice of BCS reveal important predictors such as insurance, sociodemographic characteristics, and availability of health care services. While most studies were conducted in largely single-state or limited cohorts, this contemporary study examined factors associated with BCS choice in a cohort of commercially insured females with ESBCa. Methods: This retrospective observational study of the IBM $^{\circledR}$ MarketScan ${ }^{\circledR}$ claims data spanned $01 / 01 / 2012$ to $03 / 31 / 2018$. Eligibility criteria included: 1) diagnosis of invasive breast cancer, 2) absence of metastatic or other primary/secondary cancers, and 3) BCS or mastectomy within 6 months of initial diagnosis. Predictors of BCS were examined using logistic regression, which included: 1) patient-level factors of age, BRCA $1 / 2$ testing, insurance plan type, and comorbid conditions, 2) community-level (ZIP3) measures from 2019 Area Health Resource Files, and 3) time effects. (See Table on page 929.) Results: A total of 57,299 patients were included. Higher BCS use (versus mastectomy) was significantly associated with: more recent year of diagnosis (OR: 1.08-1.72, $\mathrm{p}<.0001-\mathrm{p}=.02$ ), older age (OR: 1.96-2.44, $\mathrm{p}<.0001$ ), genetic testing (OR: $1.25, \mathrm{p}<.0001$ ), radiation presurgery (OR: $4.20, \mathrm{p}<.0001$ ), and higher density of physicians in specialties of medical genetics (OR: 6.50, $\mathrm{p}<.01$ ) or nuclear medicine (OR: 2.24, $\mathrm{p}=.04$ ). Lower BCS use was associated with: comorbid dementia (OR: 0.58, $\mathrm{p}=.01$ ), comorbid congestive heart failure (OR: $0.79, \mathrm{p}=.01$ ), chemotherapy pre-surgery (OR: $0.40, \mathrm{p}<.0001$ ), residing in the South, Midwest, or West (OR: 0.71-.89, $\mathrm{p}<.0001-\mathrm{p}=.01$ ), and a greater availability of either plastic surgeons (OR: $0.66, \mathrm{p}<.0001$ ) or hospitals with medical/surgical services (OR: $0.26, \mathrm{p}<0.01$ ). Few patients had radiation pre-surgery (1.25\%), but those patients had increased use of BCS. Conclusions: Choice of BCS over mastectomy increased over time. This study showed increased adoption with older age, access to and use of genetic services, preoperative radiation and decreased use with certain co-morbid conditions and the regions of the South, Midwest, or West. This real-world study contributes to informing clinical practice on the significant patientand community-level factors influencing BCS in an ESBCa cohort.
HSR20-086: Concurrent Chemoradiation Treatment Patterns for Stage III Non-Small Cell Lung Cancer in Veterans Anna Hung, PharmD, PhD, MSa,b; Kyung Min Lee, PhDc; Yanhong Li, MDa,b; Julie A. Lynch, PhD, RNc; Pradeep Poonnen, MDª, Bradley J. Hintze, PhDa; Olga V. Efimova, MD, PhDc;

Candice Yong, $\mathrm{PhD}^{\mathrm{d}}$; Brian Seal, PhD, MBAd;

Trudy Pendergraft, MSPH ${ }^{\text {; }}$; Michael Kelley, MDa,b; and Shelby D. Reed, PhDa,b

a Duke University School of Medicine, Durham, NC; ${ }^{b}$ Veterans Administration Medical Center, Durham, NC; ${ }^{c}$ Veterans Health Administration Salt Lake City Health Care System, Salt Lake City, UT; 'AstraZeneca, Gaithersburg, MD

Background: NCCN guidelines recommend concurrent chemoradiation (cCRT) as initial treatment for unresectable, stage III non-small cell lung cancer (NSCLC). The objective of this study is to evaluate cCRT rates over time and geographic region, and to identify patient and facility characteristics associated with receipt of cCRT. Methods: Electronic medical record data from the Veterans Affairs (VA) Corporate Data Warehouse and data from the VA Central Cancer Registry were used to identify veterans who were diagnosed with stage III NSCLC from 2013 to 2017. Patients survived at least 45 days post-diagnosis, did not have lung resection within 180 days, had at least two visits of VA cancer care, received chemotherapy (CT) and/or radiotherapy (RT) within 120 days of diagnosis, and had their records reviewed by a cancer registrar. cCRT was defined as initiation of CT and RT within 14 days of each other; while sequential chemoradiation (sCRT) was initiation more than 14 days apart. Generalized mixed models accounting for clustering by VA facility were used to determine factors associated with cCRT receipt compared to other treatment (sCRT, CT only, or RT only). Results: From 2013 to 2017, 3,414 veterans with stage III NSCLC met inclusion criteria. Fifty-five percent of these veterans received cCRT, $15 \%$ received sCRT, $14 \%$ received RT only, and $16 \%$ received CT only. The percentage of veterans receiving cCRT increased from $51 \%$ in 2013 to $62 \%$ in 2017. cCRT rates were highest in the Midwest (60\%) and lowest in the West (51\%). Fifty-six percent of whites received cCRT vs $53 \%$ of non-whites. Factors associated with increased odds of receipt of cCRT compared to any other treatment include white race (adjusted OR $[\mathrm{aOR}]=1.24 ; 95 \% \mathrm{CI}$ : $1.01-1.53$ ), later diagnosis year (2017 vs 2013: $\mathrm{aOR}=1.67 ; 95 \% \mathrm{CI}$ : $1.28-2.17$; 2016 vs 2013: aOR=1.36; 95\% CI: 1.06 1.75 ), and facility with on-site radiation oncology services $(\mathrm{aOR}=1.43 ; 95 \% \mathrm{CI}: 1.02-1.99)$. Factors associated with decreased odds of cCRT receipt compared to any other treatment included increasing age (aOR per 10 years $=0.66$; $95 \%$ CI: $0.59-0.74$ ) and Charlson-Deyo comorbidity score $(\mathrm{aOR}=0.94$; $95 \% \mathrm{CI}$ : 0.91-0.97). Conclusions: Receipt of cCRT in veterans with unresectable, stage III NSCLC slightly increased from 2013 to 2017, and 


\section{HSR20-085 Table 1. Logistic Model of Receiving Breast Conserving Surgery versus Mastectomy $(\mathrm{N}=\mathbf{5 7 , 2 9 9 )}$}

\begin{tabular}{|c|c|c|c|}
\hline Variable & $\begin{array}{l}\text { Odds } \\
\text { Ratio }\end{array}$ & $P$ Value & $\begin{array}{c}\text { 95\% Confidence } \\
\text { Interval }\end{array}$ \\
\hline
\end{tabular}

\begin{tabular}{|llll|}
\hline Patient-Level Factors & & & \\
\hline Year (Ref=2012) & & & \\
\hline 2013 & 1.08 & 0.02 & {$[1.01,1.14]$} \\
\hline 2014 & 1.14 & $<.0001$ & {$[1.07,1.22]$} \\
\hline 2015 & 1.35 & $<.0001$ & {$[1.26,1.45]$} \\
\hline 2016 & 1.69 & $<.0001$ & {$[1.57,1.83]$} \\
\hline 2017 & 1.72 & $<.0001$ & {$[1.58,1.87]$}
\end{tabular}

Age $($ Ref $=$ Ages under 50$)$

\begin{tabular}{llll}
\hline $50-59$ & 1.96 & $<.0001$ & {$[1.87,2.04]$} \\
\hline $60-69$ & 2.64 & $<.0001$ & {$[2.50,2.79]$} \\
\hline $70-79$ & 2.87 & $<.0001$ & {$[2.67,3.09]$} \\
\hline Age $80+$ & 2.44 & $<.0001$ & {$[2.20,2.70]$}
\end{tabular}

\section{Data Supplier}

$\begin{array}{llll}\text { Employer-Sponsored (versus } & 1.01 & 0.87 & {[0.94,1.07]}\end{array}$ Health Plan)

\section{Policy Holder}

Policyholder (versus Spouse or $1.06<0.01 \quad[1.02,1.10]$ Other Dependent)

\section{Region (Ref $=$ Northeast)}

\begin{tabular}{llll} 
Midwest & 0.89 & 0.01 & {$[0.81,0.97]$} \\
South & 0.71 & $<.0001$ & {$[0.64,0.78]$} \\
\hline West & 0.87 & 0.01 & {$[0.78,0.96]$}
\end{tabular}

\section{Play Type (Ref=PPO/POS)}

$\begin{array}{llll}\text { EPO or HMO } & 1.07 & 0.05 & {[1.00,1.15]} \\ \text { CDHP } & 0.99 & 0.85 & {[0.93,1.06]} \\ \text { HDHP } & 0.97 & 0.45 & {[0.89,1.05]}\end{array}$

\section{Genetic Testing}

\begin{tabular}{|c|c|c|c|}
\hline BRCA1/BRCA2 Testing & 1.25 & $<.0001$ & {$[1.18,1.32]$} \\
\hline \multicolumn{4}{|l|}{ Insitu Diagnosis } \\
\hline Also Had In Situ on Index Date & 1.06 & 0.03 & {$[1.01,1.11]$} \\
\hline Also Had In Situ pre-Index Date & 0.82 & $<.0001$ & {$[0.79,0.86]$} \\
\hline \multicolumn{4}{|l|}{ Treatment Prior to Surgery } \\
\hline Had Prior Chemotherapy & 0.40 & $<.0001$ & {$[0.37,0.44]$} \\
\hline Had Prior Radiation Therapy & 4.20 & $<.0001$ & {$[3.10,5.68]$} \\
\hline \multicolumn{4}{|l|}{ Comorbid Conditions } \\
\hline Congestive Heart Failure & 0.79 & 0.01 & {$[0.66,0.94]$} \\
\hline $\begin{array}{l}\text { Chronic Obstructive Pulmonary } \\
\text { Disease }\end{array}$ & 1.03 & 0.44 & {$[0.96,1.10]$} \\
\hline
\end{tabular}

\begin{tabular}{|c|c|c|c|}
\hline \multicolumn{4}{|c|}{$\begin{array}{l}\text { HSR20-085 Table 1. Logistic Model of Receiving } \\
\text { Breast Conserving Surgery } \\
\text { versus Mastectomy } \\
(\mathrm{N}=57,299) \text { (cont.) }\end{array}$} \\
\hline Variable & $\begin{array}{l}\text { Odds } \\
\text { Ratio }\end{array}$ & $P$ Value & $\begin{array}{l}\text { 95\% Confidence } \\
\text { Interval }\end{array}$ \\
\hline Cerebrovascular Disease & 0.93 & 0.24 & {$[0.82,1.05]$} \\
\hline Dementia & 0.58 & 0.01 & {$[0.38,0.87]$} \\
\hline Diabetes & 1.03 & 0.39 & {$[0.96,1.10]$} \\
\hline Diabetes + Complications & 1.08 & 0.34 & {$[0.92,1.26]$} \\
\hline AIDS & 0.59 & 0.14 & {$[0.29,1.18]$} \\
\hline Hemiplegia or Paraplegia & 1.78 & 0.13 & {$[0.85,3.74]$} \\
\hline Mild Liver Disease & 0.80 & 0.36 & {$[0.50,1.29]$} \\
\hline Moderate/Severe Liver Disease & 0.90 & 0.81 & {$[0.39,2.09]$} \\
\hline Acute Myocardial Infarction & 0.99 & 0.98 & {$[0.72,1.37]$} \\
\hline Peptic Ulcer & 0.94 & 0.73 & {$[0.68,1.31]$} \\
\hline Peripheral Vascular Disease & 1.09 & 0.39 & {$[0.90,1.31]$} \\
\hline Renal Disease & 1.12 & 0.11 & {$[0.97,1.29]$} \\
\hline Rheumatoid Disease & 0.94 & 0.49 & {$[0.80,1.11]$} \\
\hline \multicolumn{4}{|l|}{ Community-Level Factors } \\
\hline \multicolumn{4}{|l|}{ ZIP3-Level Variables } \\
\hline Percent Urban & 1.05 & 0.68 & {$[0.82,1.36]$} \\
\hline Percent Black & 0.98 & 0.91 & {$[0.65,1.47]$} \\
\hline Percent Asian & 0.76 & 0.44 & {$[0.39,1.52]$} \\
\hline Percent Hispanic & 0.79 & 0.11 & {$[0.59,1.05]$} \\
\hline Percent Other Race/Ethnicity & 1.82 & 0.35 & {$[0.52,6.36]$} \\
\hline $\begin{array}{l}\text { Percent with 4-Year College } \\
\text { Degree }\end{array}$ & 1.32 & 0.38 & {$[0.71,2.45]$} \\
\hline $\begin{array}{l}\text { Median Household Income (\$10 } \\
\text { thousands) }\end{array}$ & 1.01 & 0.65 & {$[0.97,1.06]$} \\
\hline $\begin{array}{l}\text { \# Ob-Gyn Physicians (per 10k } \\
\text { residents) }\end{array}$ & 1.09 & 0.03 & {$[1.01,1.18]$} \\
\hline $\begin{array}{l}\text { \# Plastic Surgery Physicians (per } \\
\text { 10k residents) }\end{array}$ & 0.66 & $<0.001$ & {$[0.53,0.81]$} \\
\hline $\begin{array}{l}\text { \# Diagnostic Radiology } \\
\text { Physicians (per 10k residents) }\end{array}$ & 0.90 & 0.04 & {$[0.81,0.99]$} \\
\hline $\begin{array}{l}\text { \# Medical Genetics Physicians } \\
\text { (per 10k residents) }\end{array}$ & 6.50 & $<0.01$ & {$[1.80,23.50]$} \\
\hline $\begin{array}{l}\text { \# Nuclear Medicine Physicians } \\
\text { (per 10k residents) }\end{array}$ & 2.24 & 0.04 & {$[1.06,4.71]$} \\
\hline $\begin{array}{l}\text { \# Radiation Oncology } \\
\text { Physicians (per 10k residents) }\end{array}$ & 1.00 & 0.99 & {$[0.65,1.54]$} \\
\hline $\begin{array}{l}\text { \# Hospitals with General } \\
\text { Medicine/Surgical Center (per } \\
\text { 10k residents) }\end{array}$ & 0.26 & $<0.01$ & {$[0.11,0.64]$} \\
\hline $\begin{array}{l}\text { \# Hospitals with Chemotherapy } \\
\text { (per 10k residents) }\end{array}$ & 1.27 & 0.64 & {$[0.47,3.43]$} \\
\hline Constant & 0.87 & 0.38 & {$[0.64,1.19]$} \\
\hline
\end{tabular}

Note: $p$-values for ZIP3-level variables are based on clustered standard errors. 
varied across Census region and facility. Veterans who were older, non-white, had more comorbidities, or were seen at a facility without on-site radiation oncology services were less likely to receive cCRT.

\section{HSR20-088: Clinical Impact of Adherence to NCCN} Biomarker Testing Guidelines on Survival for Patients With Advanced Non-Squamous Non-Small-Cell Lung Cancer Ani John, BSN, MPH, PhD; Baiyu Yang, PhD; Jaya Madala, MS; and Roma Shah, MPH

Roche Diagnostics, Pleasanton, CA

Background: Identification of molecular alterations has become a key component in the management of advanced non-small cell lung cancer (aNSCLC) patients, because these biomarkers can provide essential information to guide treatment selection. The National Comprehensive Cancer Network (NCCN) guideline recommends molecular diagnostic testing as part of the pathologic evaluation for non-squamous aNSCLC. However, in routine oncology practice, not all eligible patients are tested. Few studies have examined the impact of adherence to testing on patient outcomes in a real-world setting. Methods: Patients diagnosed with non-squamous aNSCLC (stage IIIB and above) between 2011 and 2019 from the de-identified nationwide Flatiron Health electronic health record-derived database were included in this analysis. Testing status was defined as follows: tested patients had any of the NCCN recommended molecular test (EGFR, ALK, ROS1, KRAS, BRAF, or PD-L1) any time between 14 days prior to and 90 days after aNSCLC diagnosis date; patients with no evidence of testing did not have documentation of receipt of any above-mentioned tests during this time period. Median survival was calculated using Kaplan-Meier analysis. Unadjusted and adjusted Cox proportional hazards regression models were used to evaluate the association between testing status and overall survival. Results: A total of 29,658 eligible patients were included $(75.3 \%$ tested; $24.7 \%$ no evidence of testing). Mean age at diagnosis was 69 years (SD: 15 years). The majority of patients (90\%) were from the community clinics, and $31 \%$ of patients had commercial insurance plans. Compared to tested patients, patients with no evidence of testing were less likely to be enrolled in a commercial health plan, and more likely to have a history of smoking or be diagnosed before 2013 ( $p<$ 0.05 ). Tested patients had a lower risk of mortality in the unadjusted analysis (hazard ratio 0.84, 95\% CI 0.82-0.87) and lived longer than patients with no evidence of testing (median survival 17.1 months [95\% CI 16.8 -17.5] vs. 13.8 months [95\% CI 13.2-14.4]), and the association remained significant after adjusting for potential confounders including age at diagnosis, sex, history of smoking, initial stage at diagnosis, race, and year of advanced diagnosis (hazard ratio 0.77, 95\% CI 0.74-0.79). Conclusions: Among non-squamous aNSCLC patients, following the NCCN biomarker testing guidelines may be associated with more favorable survival outcomes.

HSR20-089: Association of Patient Clinico-Genomic Characteristics With Tumor Mutational Burden in Small Cell Lung Cancer: An Observational Study

Sumesh Kachroo, $\mathrm{PhD}^{\mathrm{a}}$; Changxia Shao, $\mathrm{PhD}^{\mathrm{b}}$; Kaushal Desai, $\mathrm{PhD}^{\mathrm{c}}$; Jinghua $\mathrm{He}, \mathrm{PhD}^{\mathrm{b}}$; Fan Jin, $\mathrm{MD}^{\mathrm{b}}$; and Shuvayu Sen, $\mathrm{PhD}^{\mathrm{c}}$

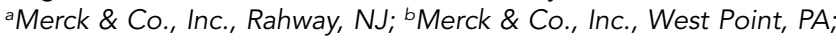
cMerck \& Co., Inc., Kenilworth, NJ

Background: This study evaluated the relationship between patients' clinical and genomic characteristics and high tumor mutational burden (TMB) in the context of small cell lung cancer (SCLC). Methods: This was a retrospective cohort study using the deidentified Flatiron Health-Foundation Medicine Clinico-Genomic Database linking clinical electronic health records and comprehensive genomic profiling (CGP) data from January 2011-September 2018. Patients $\geq 18$ years with confirmed SCLC pathology, FoundationOne ${ }^{\circledR}$ genomic profiling data, and chemotherapy treatment were included. We examined treatment patterns, genomic alterations, TMB score, survival and the association of clinical and genomic variables with high TMB as determined a by multivariate logistic regression. High TMB (TMB-H) was defined as $\geq 10$ mutations/megabase (mut/Mb). Results: A total of 186 patients were included in the study. CGP test results were reported prior to initiation of first-line (1L) treatment in $6.9 \%$ of patients and prior to second-line (2L) treatment in $65.6 \%$ of patients. $1 \mathrm{~L}$ and $2 \mathrm{~L}$ treatment patterns were similar for patients with high and low TMB scores. The most frequent $1 \mathrm{~L}$ therapy was a combination of carboplatin and etoposide $65.3 \%$ in TMB-H patients, $67.3 \%$ in non TMB-H patients). A total of 95 patients continued to $2 \mathrm{~L}$ therapy, and topotecan monotherapy was the most frequent $2 \mathrm{~L}$ therapy $(12.0 \%$ in TMB-H patients, $15.4 \%$ in non TMB-H patients). The median duration of $1 \mathrm{~L}$ and $2 \mathrm{~L}$ treatment in TMB-H and non TMB-H patients were 11.1 vs. 7.9 months for $1 \mathrm{~L}$ and 7.9 vs. 5.6 months for $2 \mathrm{~L}$ (adjusted $\mathrm{P}$ values of 0.76 and 0.28 respectively). The most frequent short variant mutations (including variants of unknown significance) observed were in TP53(90.86\%), RB1(65.05\%), LRP1B(32.79\%), and FAT3(20.96\%). Loss of heterozygosity in TP53 was confirmed in $62.4 \%$ of patients and high microsatellite instability was recorded in $0.7 \%$ of patients tested. TMB score was determined for 179 patients. The mean (SD) TMB score was 9.75 (7.89), the median (IQR) TMB score was 8.70 (5.2-13.0), and 41.9\% had a TMB score $\geq 10$. Variables significantly associated with a high TMB score at $\mathrm{P} \leq 0.05$ were short variants of LRPB1, FAT3, MLL3, MED12, and NOTCH3. The median overall survival from time of diagnosis was 14.9 months in patients with high TMB and 14.7 months in those with low TMB, with an adjusted hazard ratio 0.985 (95\% CI 
0.648, 1.498). Conclusions: Treatment patterns and survival did not differ by TMB status.

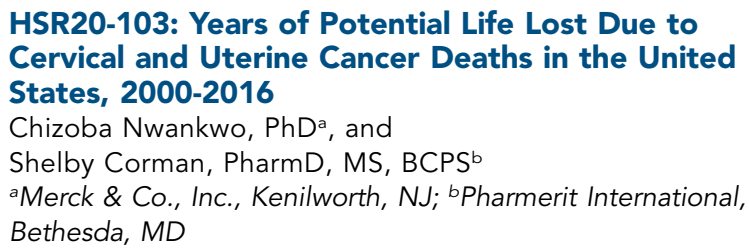

Introduction: Early death due to cancer results in lost societal productivity and is an important contributor to the overall burden of the disease. The objective of this study was to examine trends in years of potential life lost (YoPLL) due to cervical and uterine cancer in the United States between 2000 and 2016. Methods: YoPLL due to cervical and uterine cancer in each year was calculated by summing, for each death, the remaining life expectancy at the age of death. The number of deaths by age due to each cancer type and remaining life expectancy by age were obtained from the World Health Organization (WHO) Mortality Database and Life Tables, respectively. YoPLL is presented as the sum across all deaths due to cervical or uterine cancer in the US each year, and as the average YoPLL per death, calculated as the total YoPLL divided by the number of deaths in each year. Results: The number of cervical cancer deaths per year was relatively stable during the study period, lowest in 2004 (3,847 deaths) and highest in 2016 (4,419 deaths). Total YoPLL and average YoPLL per death followed a similar pattern, ranging from 107,600 to 117,590 YoPLL per year (Figure) and 26.5 to 28.7 YoPLL per death. Conversely, the number of uterine cancer deaths per year increased from 6,570 in the year 2000 to 10,713 in 2016, and total YoPLL increased from 110,266 to 207,684 (Figure). The average YoPLL per uterine cancer death increased from 16.8 in 2000 to 19.4 in 2016. Conclusions: YoPLL is a measure of the societal burden of early death and is a key component of the global burden of cancer. Between 2000 and 2016, YoPLL due to uterine cancer nearly doubled, while YoPLL due to cervical cancer remained stable.

\section{HSR20-112: Quantifying Caregiver Preferences for Attributes Associated With First-Line Treatment of Metastatic Non-Small Cell Lung Cancer \\ Candice Yong, $\mathrm{PhD}^{\mathrm{a}}$; Brian Seal, RPh, MBA, PhD; M. Janelle Cambron-Mellott, $\mathrm{PhD}^{\mathrm{b}}$; Oliver Will, $\mathrm{PhD}^{\mathrm{b}}$; Martine C. Maculaitis, $\mathrm{PhD}^{\mathrm{b}}$; Kelly Clapp, $\mathrm{PhD}^{\mathrm{b}}$; Emily Mulvihill, MBA Ion Cotarla, MD, PhDa; and Ranee Mehra, $\mathrm{MD}^{c}$ \\ a AstraZeneca, Gaithersburg, MD; ${ }^{b}$ Kantar, New York, NY; ' University of Maryland Marlene and Stewart Greenebaum Cancer Center, Baltimore, $M D$}

Background: As caregivers (CGs) may play a key role in helping patients manage clinical and non-clinical aspects of treatment for metastatic non-small cell lung cancer (mNSCLC), it is useful to understand their perceptions of the benefits and risks of currently available therapies and how they value the different efficacy and toxicity profiles of novel therapies. This study quantified CG preferences for attributes associated with chemotherapy and immunotherapy, alone or in combination, for mNSCLC and the economic burden of treatment. Methods: CGs of patients with mNSCLC completed an online, cross-sectional survey assessing preferences via a discrete choice experiment (DCE), which was designed based on qualitative research with CGs and pilot tested. In the DCE, 2 hypothetical treatment profiles, varying on 7 attributes (Table 1) were presented side-by-side in a series of tasks, and CGs chose their preferred option. Hierarchical Bayesian modeling was used to estimate preference weights for each attribute level. Additional questions inquired about economic burden. Results: CGs ( $\mathrm{N}=166 ; 67 \%$ female) provided care a mean of 36 hours per week, usually for a spouse $(65 \%)$. Almost half were

HSR20-103 Figure: Total Years of Potential Life Lost due to Cervical and Uterine Cancers in the United States (2000-2016)

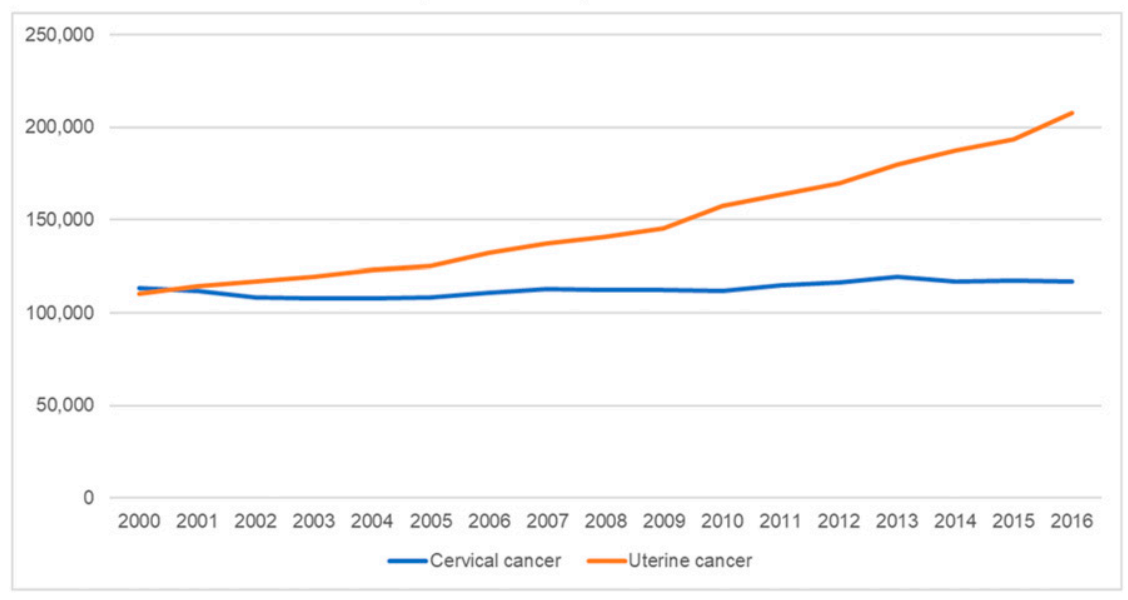


HSR20-112 Table 1. Attributes and levels associated with 1L treatment included in the DCE

\begin{tabular}{|c|c|c|}
\hline Attribute & Attribute wording & Level \\
\hline \multirow[t]{3}{*}{ Median overall survival } & \multirow[t]{3}{*}{ Survival (life expectancy) of $X$ months } & 11 months \\
\hline & & 17 months \\
\hline & & 30 months \\
\hline \multirow[t]{3}{*}{ Median progression-free survival } & \multirow[t]{3}{*}{ Remain stable (cancer does not worsen) for $\mathrm{X}$ months } & 5 months \\
\hline & & 8 months \\
\hline & & 10 months \\
\hline \multirow[t]{3}{*}{ Nausea (all grades) } & \multirow{3}{*}{$\begin{array}{l}\text { X\% risk of nausea affecting appetite, which could } \\
\text { lead to dehydration and malnutrition }\end{array}$} & $69 \%$ \\
\hline & & $36 \%$ \\
\hline & & $10 \%$ \\
\hline \multirow[t]{3}{*}{ Pneumonitis (all grades) } & \multirow{3}{*}{$\begin{array}{l}\mathrm{X} \% \text { risk of inflammation in the lungs, which could } \\
\text { become serious and possibly require temporary } \\
\text { medical oxygen }\end{array}$} & $8 \%$ \\
\hline & & $5 \%$ \\
\hline & & $<1 \%$ \\
\hline \multirow[t]{3}{*}{ Skin rash (all grades) } & \multirow{3}{*}{$\begin{array}{l}\text { X\% risk of skin rash involving itching and/or burning, } \\
\text { which could become severe, limiting daily activities }\end{array}$} & $22 \%$ \\
\hline & & $17 \%$ \\
\hline & & $12 \%$ \\
\hline \multirow[t]{3}{*}{ Grade 3/4 AE } & \multirow{3}{*}{$\begin{array}{l}\mathrm{X} \% \text { risk of a serious side effect that may lead to } \\
\text { hospitalization }\end{array}$} & $70 \%$ \\
\hline & & $48 \%$ \\
\hline & & $18 \%$ \\
\hline \multirow[t]{4}{*}{ Regimen } & \multicolumn{2}{|c|}{$\begin{array}{l}2 \text { to } 8 \text { hours of chair time receiving IV therapy every } 1 \text { to } 3 \text { weeks for } 3 \text { to } 4 \text { months then } 45 \text { to } 75 \\
\text { minutes every } 3 \text { to } 4 \text { weeks }\end{array}$} \\
\hline & \multicolumn{2}{|c|}{1 to 4 hours of chair time receiving IV therapy every 1 to 3 weeks } \\
\hline & \multicolumn{2}{|c|}{$\begin{array}{l}1 \text { to } 2 \text { hours of chair time receiving IV therapy every } 3 \text { to } 4 \text { weeks for } 3 \text { to } 4 \text { months then } 30 \text { to } 60 \\
\text { minutes every } 2-4 \text { weeks }\end{array}$} \\
\hline & 30 to 60 minutes of chair time receiving IV therapy e & \\
\hline
\end{tabular}

Note. AE: adverse event; IV: intravenous.

currently employed. Increasing OS from 11 to 30 months was most important; it was $5.5 \%$ more important than decreasing the risk of a serious adverse event that may lead to hospitalization (Grade 3/4 AE) from $70 \%$ to $18 \%$. These attributes were over twice as important as the other attributes (Figure 1). To accept an increase in risk of a Grade 3/4 $\mathrm{AE}$ from $18 \%$ to $70 \%$, all grades nausea from $10 \%$ to $69 \%$, skin rash from $12 \%$ to $22 \%$, and pneumonitis from $<1 \%$ to $8 \%$, OS would need to increase by $17,4,1.5$, and 0.4 months, respectively. Most CGs (72\%) provided financial support; $30 \%$ incurred out-of-pocket (OOP) costs for travel to treatment, and $25 \%$ reported unpaid leave from work. Among CGs who reported OOP costs, 19\%, 28\%, 53\%, and $57 \%$ rated costs associated with cancer drugs, travel, lodging, and unpaid leave, respectively, as burdensome (rating of 4 or 5 on a 5-point scale). Conclusions: The results showed that to accept a higher toxicity risk, CGs may require up to 1.5 years of increased OS in exchange. Findings also suggest that treatment for mNSCLC poses an economic burden for most CGs in terms of both direct and indirect (e.g., unpaid leave) costs. Treatment selection in 1L mNSCLC that considers the CG role should be informed by benefits/risks of treatment, as well as cost factors.
HSR20-113: Diabetes Complications and Risks of Breast Cancer Recurrence Among Older Women

Jifang Zhou, MD, MPHa; Alemseged A. Asfaw, MSa;

Nadia A. Nabulsi, MPHa; Nita A. Mukand, BSa; Inyoung Lee, MSa;

Naomi Y. Ko, MD, MPH, AM ${ }^{\text {b }}$, Denise M. Boudreau, PhD, RPh ${ }^{c, d}$; and Gregory S. Calip, PharmD, MPH, PhDa,e

aniversity of Illinois at Chicago, Chicago, IL; ${ }^{b}$ Boston University School of Medicine, Boston, MA; ${ }^{~} K$ Kaiser Permanente Washington Health Research Institute, Seattle, WA; dUniversity of Washington, Seattle, WA; ' University of Illinois Cancer Center, Chicago, IL

Background: Type 2 diabetes mellitus (DM) is associated with increased breast cancer (BC) risk and adverse BC outcomes. Less is known about the impacts of diabetes severity and its complications on $\mathrm{BC}$ recurrence. Our objective was to determine risk of recurrence associated with DM complications among older women with invasive BC. Methods: We conducted a retrospective cohort study of women ages $65+$ years that were diagnosed with incident, invasive stages I-III BC between 2007 and 2015 using the Surveillance, Epidemiology and End Results (SEER)Medicare Linked Database. SEER records and administrative health claims data were used to ascertain patient demographics, tumor characteristics and cancer treatment including chemotherapy, surgery and radiation. Claims data 
were used to estimate the prevalence of DM complications and calculate baseline and time-varying Diabetes Complications Severity Index (DCSI) scores. BC recurrences were identified using a validated algorithm for health claims data. Multivariable competing risk models accounting for death without $\mathrm{BC}$ recurrence were used to estimate adjusted subdistribution hazards ratios (SHR) and 95\% confidence intervals (CI) for associations between DCSI scores, specific DM complications and recurrence risks. Results: Overall, 12,628 women ages $65+$ years with DM at BC diagnosis were included. Over a median follow-up of 61 months, 2,134 (17\%) women experienced BC recurrence. More women that developed a recurrence had DCSI scores $\geq 2$ compared to women with DCSI score $<2$ ( $58 \%$ vs. $52 \%$ ). In multivariable models, women with DCSI scores $\geq 3$ (SHR 1.22; 95\%CI 1.08-1.37) and 2 (SHR 1.15; 95\%CI 1.01-1.30) had increased risks of recurrence compared to women with scores of 0. Specific macrovascular (cardiovascular disease SHR 1.10; 95\% CI 1.00-1.20) and microvascular (neuropathy SHR 1.17; 95\% CI 1.06-1.29) complications were independently associated with $\mathrm{BC}$ recurrence. Conclusions: We observed greater risk of $\mathrm{BC}$ recurrence in women with evidence of more severe DM and presence of macro- and microvascular complications. This association may be related to the roles of DM in $\mathrm{BC}$ pathogenesis through actions of insulin resistance and hyperinsulinemia on insulin-like growth factor receptors, or possibly the impacts of higher DM severity on BC treatment decision-making. Further research is warranted to investigate whether DM control and newer generation DM medications with cardiovascular benefits may improve breast cancer outcomes.

\section{QUALITY IMPROVEMENT}

QIM20-136: Integration of Tobacco Treatment Into Cancer Care at Stanford Health Care

Cindy Tran, MPHa; Brittany Pike, MS, BSN, RNa;

Matthew Kendra, $\mathrm{PhD}^{\mathrm{b}}$; Rachelle Mirkin, $\mathrm{MPH}^{\mathrm{a}}$;

and Judith J. Prochaska, $\mathrm{PhD}, \mathrm{MPH}^{\mathrm{c}}$

aStanford Health Care, Palo Alto, CA; 'bStanford University School of Medicine, Stanford, CA; 'Stanford University, Stanford, CA

Background: Tobacco is the leading cause of cancer and cancer-related deaths in the United States. The Stanford
Cancer Center was awarded a Moonshot P30 Supplement to integrate evidence-based tobacco treatment into cancer care. We have partnered with 3 clinics with high tobacco use prevalence and tobacco screening rates over $95 \%$. However, less than $10 \%$ of identified tobacco users were referred for treatment and less than $1 \%$ engaged in treatment. Aims: We aimed to (1) Increase tobacco treatment referrals so $100 \%$ of identified tobacco users are automatically referred for treatment (opt-out model) and (2) Support patients' long-term tobacco abstinence, which is assessed 6 months post-treatment. Methods: To address the gap between the number of identified tobacco users and the number referred for treatment, we developed an automated referral process whereby: (1) The medical assistants screen patients for tobacco use; (2) A visual indicator alerts providers of patients who use tobacco; and (3) Providers perform brief counseling and inform patients that a tobacco treatment team specialist will call them within 1 week. When called, patients are offered a menu of treatment options, including: (1) E-referral to the California Quit Line and smokefree.gov; (2) Counseling individually or as a family, in-person or via telemedicine, or in-person group counseling; (3) Cessation medications including nicotine replacement therapy, varenicline, or bupropion, with same-day delivery at a discounted price via Alto Pharmacy. Results: Since January 2019, 317 tobacco users were identified, and all were opted-in to phone outreach by the tobacco treatment service; $214(68 \%)$ patients were reached by phone, and 79 (37\% of those reached) engaged in treatment. To date, 7 (9\%) of patients are tobacco-free and $72(91 \%)$ are attempting to quit. Conclusion: We increased the tobacco treatment referral rate 10 -fold and treatment engagement 20 -fold. Our ongoing assessment of 6-month patient outcomes began in September. We track the cost of our services to inform a sustainable financial model. By adhering to the "Plan, Do, Study, Act" methodology, we implement weekly improvements to advance the quality and effectiveness of our tobacco treatment services. We will expand to include all Stanford Cancer Center and Health Care clinics. Funding: P30CA1244351-1S2 with a Moonshot Supplement. 